\title{
Hypercoagulability syndrome due to glycosylphosphatidylinositol deficiency
}

INSERM

\section{Source}

INSERM. (1999). Orphanet: an online rare disease and orphan drug data base.

Hypercoagulability syndrome due to glycosylphosphatidylinositol deficiency.

ORPHA:83639

The combination of a propensity for venous thrombosis and seizures has been reported in two unrelated kindreds. Transmission is autosomal recessive. It results from a point mutation of PIGM, which reduces transcription of PIGM and blocks mannosylation of glycosylphosphatidylinositol (GPI), leading to partial but severe deficiency of GPI. 